\title{
del concepto de egocentrismo
}

\section{espacial}

\section{en tareas de adopción \\ de perspectivas}

\section{Ileana Enesco *}

\section{Universidad Complutense de Madrid}

\section{INTRODUCCION}

Desde que en 1947 Piaget e Inhelder publicaran su trabajo sobre el desarrollo de la coordinación de perspectivas, incluido en La Représentation de l'espace chez lenfant, las réplicas e investigaciones basadas total o parcialmente en este estudio han proliferado hasta el punto de necesitar, cada cierto tiempo, revisiones comprensivas del tema. Exagerando un poco, podríamos decir que existen casi tantas divergencias como autores que se han ocupado de este problema. Afortunadamente, ésta es sólo una primera impresión, pues si analizamos con cierto detalle la literatura sobre el tema, veremos que tras las discrepancias teóricas se esconden mäs problemas metodológicos que epistemológicos.

En este artículo nos proponemos revisar de una forma comprensiva el amplio cuerpo de investigaciones originado a partir de la conocida prueba piagetiana de "Las Trẹs Montañas» en relación con un concepto fundamentalmente polémico, el del egocentrismo. Dicho concepto ocupa un importante lugar en la descripción piagetiana del llamado pensamiento preoperatorio. Por egocentrismo se entiende la incapacidad del niño pequeño para situarse en puntos de vista ajenos al propio. Asi definido, es obvio que las tareas que exigen una coordinación de distintas perspectivas - siendo la del sujeto tan sólo una más-, se verán entorpecidas por la presencia de esta característica cognitiva que es el egocentrismo. Sin embargo, como veremos a lo largo de este artículo, la ausencia de egocentrismo no garantiza necesariamente la solución de los problemas planteados en la coordinación de diferentes perspectivas. Así pues, nos encontramos con que el egocentrismo no es una condición suficiente para explicar el fracaso en este tipo de tareas. Uno de los objetivos de este artículo será intentar averiguar si, además de insuficiente, es un concepto inoperante en la explicación del desarrollo de esta habilidad cognitiva.

* Dirección de la autora: Universidad Complutense, Facultad de Psicologia, Dpto. Psicologia Evolutiva, Somosaguas. 28023 Madrid. 
Puesto que los aspectos metodológicos de la investigación sobre perspectivas espaciales serán el plato fuerte de este artículo, conviene que recordemos brevemente cómo estudiaron Piaget e Inhelder este problema. Estos autores plantearon a 100 niños de 4 a 12 años el problema de averiguar las distintas perspectivas de tres montañas de tamaño y color diferentes, situadas en una maqueta frente al sujeto. Una muñeca era colocada sucesivamente en torno a la maqueta desempeñando el papel de uobservadon, es decir, adoptando las perspectivas que el niño debía averiguar. Se utilizaron tres procedimientos: en el primero se le daba al niño tres cartones recortados según la forma y tamaño de cada montaña y se le pedia que reconstruyera la perspectiva que tenía la muñeca. En el segundo se le presentaban so cuadros diferentes que representaban a las montañas desde perspectivas diferentes e incluso perspectivas "falsas" o imposibles, y el niño debía elegir el que correspondía a la perspectiva de la muñeca. El tercer procedimiento consistia en darle un cuadro que representaba una perspectiva determinada y pedirle al sujeto que colocara la muñeca en la posición desde la cual tuviera esa perspectiva. Los sujetos debían reconstruir o identificar la propia perspectiva y las de 90,180 y 270 grados respecto a ellos.

Los resultados, en términos muy generales, fueron los siguientes: se observó que hasta los 4 ó $s$ años los niños eran incapaces de comprender la tarea. Durante un segundo estadio (II A y II B) las respuestas consistian en una reproducción de la propia perspectiva cualquiera que fuera la posición pedida. Piaget e Inhelder interpretaron esta conducta como una manifestación del egocentrismo infantil, es decir, la incapacidad para considerar puntos de vista ajenos al propio. Por último, el estadio III (III A y III B) se caracterizaba por una creciente coordinación y diferenciación de perspectivas que desembocaba, hacia los 9 ó io años, en un completo dominio operacional de la tarea.

El problema de la coordinación de perspectivas tenía para Piaget e Inhelder un doble interés. Por una parte, las propias nociones espaciales implicadas en él: para resolver la tarea de "Las Tres Montañas", el sujeto deberá considerar las posiciones relativas de los objetos entre sí y de cada objeto en relación a los distintos puntos de vista. Ello implica un dominio de las relaciones espaciales delante-detrás e izquierda-derecha. Por otra parte, el problema tenía el atractivo de enfrentarnos a la actitud cognitiva del niño en la adopción de perspectivas. Fue, sin duda, este segundo aspecto lo que llevó a tantos investigadores a repetir, transformar e idear nuevas experiencias basadas en esta tarea. ¿Cree realmente el niño pequeño que su punto de vista es el único posible? ¿Es el egocentrismo una actitud cognitiva general del pensamiento preoperatorio o se trata simplemente de una respuesta ligada a determinadas situaciones?

La mayoria de las discrepancias que se observan en los resultados de distintas investigaciones giran precisamente en torno a estas preguntas. Muchos autores han encontrado una proporción despreciable de respuestas egocéntricas cualquiera que sea la edad de los sujetos mientras que otros han descubierto que persisten hasta edades muy avanzadas. Estas contradicciones también se observan en relación con la edad de adquisición de la habilidad de coordinar perspectivas. En 
algunas investigaciones niños de 4 ó 5 años resuelven sin dificultad problemas de perspectivas y, en otras, la mayoria de los sujetos de 12 años sigue fracasando en este tipo de tareas.

Como puede suponerse, aunque todos estos trabajos comparten el objetivo de estudiar la evolución de la capacidad de adoptar otras perspectivas, las tareas y los métodos utilizados varian considerablemente de una investigación a otra.

En el apartado siguiente, revisaremos con detalle los aspectos metodológicos de la investigación sobre perspectivas espaciales con el fin de iluminar algunos resultados oscuros.

\section{PERSPECTIVAS METODOLOGICAS EN LA INVESTIGACION SOBRE LA REPRESENTACION ESPACIAL}

La exposición que sigue está organizada de acuerdo con las diferentes metodologías adoptadas en el estudio de las perspectivas. Tomaremos como referencia la prueba de "Las Tres Montañas" para analizar los cambios metodológicos que ha sufrido y que afectan a distintos aspectos del problema.

En general, estos cambios se observan en relación con: 1 , el tipo de material utilizado, y 2, el tipo de tarea que debe realizar el sujeto. En cuanto al material, las modificaciones más importantes son relativas a: a) el número de elementos, b) sus características que definen el grado de familiaridad, c) la naturaleza bidimensional o tridimensional de los estímulos de elección y d) la naturaleza animada o inanimada del observador. Con respecto a la tarea, ésta puede implicar:

a), Un proceso de inferencia frente a un proceso de reconocimiento, b) una identificación frente a una construcción de las perspectivas y c) un problema de carácter perceptivo frente a uno de carácter conceptual.

Algunos de estos aspectos fueron recogidos por Fehr (1978) en una interesante revisión del tema en la que intenta explicar el origen de los desfases que se observan en este tipo de tareas. Muchos autores han abordado el problema desde esta perspectiva metodológica, analizando alguna de estas variables que parecen tener efectos diferenciales en la actuación de los sujetos (Brodzinsky, et al., 1972; Coie, et al., 1973; Cox, 1975, 1978; Eiser, 1974; Flavell, et al., 1968, 1978, 1981; Nigl y Fishbein, 1974).

\section{Variables relativas al material utilizado}

En una revisión anterior (Enesco, 1982) hemos analizado exhaustivamente los posibles efectos diferenciales de cada una de las cinco variables de material. Aquí nos limitaremos a recordar las conclusiones generales a las que llegamos.

El material utilizado por Piaget e Inhelder en la tarea de «Las Tres Montañas», nuestro obligado punto de referencia, presentaba las siguientes características: a) constaba de tres elementos, b) éstos eran 
familiares (montañas), c) los estímulos de elección eran bidimensionales (dibujos de las montañas o cartones recortados) y d) el papel de «observador" lo desempeñaba un objeto inanimado (una muñeca).

Pocos son los estudios pospiagetianos que mantienen constantes estas variables. Con respecto al número de elementos, algunos autores que estudiaron el efecto de esta variable encontraron que la tarea era más sencilla con un sólo objeto que con tres (Flavell, et.al., 1968; Fishbein, et al., 1972). Sin embargo, otros no observaron diferencias de dificultad debidas al número de elementos (Brodzinsky, et al., 1972; Minnigerode y Carey, 1974; Nigl y Fishbein, 1974; Borke, 1975). Probablemente, sea el estudio de Phinney y Nummedal (1979) el que más luz aporta a este problema. Al estudiar la actuación de los sujetos con uno y dos objetos observaron que las dificultades dependian del tipo de relaciones espaciales que debían considerar y no del número de elementos. Por ejemplo, cuando la tarea consistía en realizar una inversión "delante-detrás" de un sólo objeto, el éxito era precoz y mayoritario desde la edad preescolar. Sin embargo, la inversión derecha-izquierda de un objeto (un perfil humano) resultó tan difícil como una inversión del mismo tipo entre dos objetos y más difícil que la inversión de dos objetos simétricos (una mesa y un árbol). Con los datos que se poseen actualmente sobre el efecto de esta variable, se puede esperar que un mismo número de elementos represente distintos niveles de dificultad $\mathrm{y}$, a la inversa, un número diferente de objetos presente dificultades semejantes. Las diferencias deben atribuirse, pues, a la influencia de otras variables, como el tipo de relaciones espaciales que el sujeto tiene que considerar (siendo la relación izquierda-derecha la de mayor dificultad), la simetría-asimetría de los objetos, etc.

El grado de familiaridad parece ser una variable más determinante que la anterior. En las tareas de coordinación de perspectivas se han utilizado desde objetos "raros» y «desconocidos» para el niño, como figuras irregulares no asimilables a ningún objeto concreto (Flavell, 1981), hasta juguetes que representan personajes de ficción del mundo infantil, como los de Walt Disney, Sesame Street, etc. (Borke, 1975). Los autores que han utilizado objetos poco o nada familiares, entre los que se incluyen figuras geométricas, como cilindros, conos, etc., encuentran un porcentaje muy elevado de errores en sujetos de hasta I6 años de edad (Flavell, et al., 1968; Laurendeau y Pinard, 1970; Garner y Plant, 1972). Por el contrario, en los estudios que se han servido de juguetes y personajes de ficción infantiles se observa que niños de 4 y 5 años resuelven sin grandes dificultades la tarea y que las respuestas egocéntricas son muy escasas a partir de los 4 años.

Sin embargo, seria engañoso concluir que el grado de familiaridad explica todas las dificultades en la coordinación de las perspectivas de los objetos. Los estudios que acabamos de citar no son todos ellos comparables respecto a la variable familiaridad, pues, en algunos casos, se modifican también otras condiciones de la tarea.

Así, por ejemplo, Borke, además de utilizar objetos muy familiares para los niños, simplificó la tarea pidiéndoles sencillamente que identificaran la perspectiva correcta, es decir, convirtió la tarea en un simple problema de reconocimiento sin que mediara ningún proceso inferencial. Por el contrario, los resultados de Laurendeau y Pinard y los de Flavell son mucho más instructivos, puesto que ambos utilizan un 
procedimiento similar al de Piagét e Inhelder y sólo modifican la variable de familiaridad de los elementos del modelo. A partir de estos resultados, podemos afirmar con mayor seguridad que la familiaridad de los objetos facilita la resolución de la tarea. Es probable que esto se deba al mayor número de rasgos diferenciadores que poseen los objetos familiares, lo que permite discriminar con mayor facilidad sus posiciones y orientaciones relativas.

La naturaleza bidimensional o tridimensional de los estímulos para la construcción o identificación de las perspectivas ha sido una variable menos estudiada que las anteriores. Por lo general, se han empleado estímulos bidimensionales más o menos semejantes a los de Piaget e Inhelder (1948), que consisten en fotos, dibujos o recortes que representan distintas perspectivas del modelo. En estos casos, el sujeto pasa de la percepción de un modelo tridimensional a la identificación (mediante fotos o dibujos) o construcción (mediante recortes) de una perspectiva representada bidimensionalmente.

La interpretación tridimensional de un plano implica una serie de habilidades perceptivas que dependen de la edad, la práctica, el medio cultural, etc. (Hudson, 1962; Deregowski, 1971) y parece comprobado que resulta más difícil interpretar estimulos bidimensionales que tridimensionales (Gibson, 1969). En los primeros, los rasgos distintivos son menos sobresalientes $y$, en consecuencia, para obtener la misma información que se obtiene de objetos tridimensionales, la exploración visual debe ser mucho más exhaustiva. Es bien sabido que los sujetos más jóvenes suelen presentar conductas de exploración - visual o táctil- mucho más pobres que los mayores y que una exploración deficiente puede conducir a la elección de un estímulo incorrecto en la tarea de perspectivas. Además, los niños pequeños poseen menor experiencia en la interpretación de fotos, dibujos, etc. Es probable, pues, que estos factores, presentes en las tareas de perspectivas que emplean un modelo tridimensional y estímulos bidimensionales, interfieran en su resolución. Deberiamos preguntarnos, pues, si las tareas que emplean este tipo de material no están midiendo alguna otra destreza además de la habilidad para coordinar perspectivas, como sugiere Fehr (1978).

Para poner a prueba la hipótesis de que los estimulos bidimensionales entorpecen la resolución del problema de perspectivas, Nigl y Fishbein (1974) compararon la actuación de los niños con este tipo de estímulos (fotos) y con objetos tridimensionales idénticos a los del modelo. Encontraron que hasta los 7 años, los niños tenian dificultades incluso en la identificación de la propia perspectiva cuando tenían que realizarla a partir de fotos. Para todos los sujetos fue más sencillo trabajar con objetos idénticos al modelo cuando se trataba de reconocer su propio punto de vista. En la identificación de otras perspectivas ( 90 , I 80 y 270 grados), el efecto facilitador de los estímulos tridimensionales se observó sólo en el grupo de edad mayor (10 años), lo que confirma el carácter evolutivo de esta habilidad.

En conclusión, parece pues que la utilización de objetos tridimensionales facilita $o$, si se quiere, no entorpecè la puesta en práctica de la habilidad para coordinar perspectivas siempre que, eso sí, esta capacidad esté ya presente: 
Por último, sobre los efectos de la naturaleza animada o inanimada del "observador", es decir, del sujeto u objeto que se desplaza en torno al modelo colocándose en distintas perspectivas, contamos aún con poca información. Cox (1975) estudió esta variable y encontró que los niños (de 6 y 7 años) que tenían que imaginarse la perspectiva de una muñeca cometian más errores egocéntricos que los que debian imaginarse la de un sujeto humano real. Sin embargo, en otro trabajo, Borke (1975), utilizando un personaje de ficción como "observador" que viajaba alrededor del modelo, encontró que los niños de 3 y 4 años eran capaces de comprender la perspectiva de otra "persona». La historia y, en general, el problema se planteaban como un juego para el niño y se le insistía que el muñeco iba a jugar a ese «juego».

Es probable que estos resultados diferentes se deban a la forma como se presentaba la tarea en cada caso. Borke la presentaba como una ficción y no como un problema, mientras que Cox preguntaba directamente a los niños qué perspectiva tenía la muñeca introduciendo la tarea con un carácter de obligatoriedad y no como una actividad lúdica. Es posible que en este último caso los niños se sientan muy desconcertados al plantearles una actividad intelectual que tiene un componente de ficción tan llamativo (el de una muñeca "adoptando» distintas perspectivas o haciendo fotos de un paisaje). A este respecto, son sumamente interesantes las observaciones de Elkonin (1978) que muestran cómo en determinadas condiciones de juego experimental los niños logran superar su egocentrismo cognitivo y solucionar problemas que, en otras condiciones, suelen provocar respuestas egocéntricas.

\section{Variables de tarea}

Es difícil encontrar más de dos trabajos que hayan utilizado los mismos procedimientos con variaciones en algún aspecto del material o, a la inversa, el mismo material con procedimientos distintos. Desde luego, esto constituye un problema a la hora de averiguar el papel de cada variable. Si las condiciones del material parecen afectar en mayor o menor medida la actuación de los sujetos, las condiciones de la tarea no serán menos responsables de la facilidad o dificultad de los sujetos para resolverla, como veremos ahora.

\section{Segun el procedimiento: ¿inferencia frente a reconocimiento?}

En las tareas de perspectivas se han utilizado básicamente dos procedimientos que difieren según la información que tiene el sujeto sobre el modelo cuyas perspectivas debe predecir. En uno de estos procedimientos, el sujeto, ocupando siempre una misma posición respecto al modelo, debe predecir sus distintas perspectivas sin haber tenido la oportunidad de desplazarse previamente en torno a él. En el segundo procedimiento, el sujeto puede observar el modelo desde distintas posiciones antes de predecir los puntos de vista requeridos. Asi como el primer procedimiento no proporciona ninguna posibilidad de "aprender" o memorizar las distintas perspectivas del modelo, en el segundo no podemos descartar la intervención de estos procesos. Además, la oportunidad de desplazarse alrededor del modelo proporciona al sujeto la posibilidad de observar que un cambio de posición 
puede implicar un cambio en la apariencia del modelo. Por el cuntrario, en la primera condición, el sujeto debe no sólo inferir cómo es la perspectiva, sino también comprender que puede ser distinta.

Aun cuando es muy improbable que bajo la segunda condición la tarea se resuelva por "memorización» de las distintas perspectivas, la mediación del recuerdo - y de la información que proporciona esta condición - puede explicar parte de las discrepancias que existen entre los resultados obtenidos con uno y otro procedimiento. Por ello, y siguiendo a Eiser (1974), hablaremos de dos tipos de procesos según el procedimiento:

I. Un proceso inferencial, cuando el sujeto debe predecir las perspectivas del modelo sin haber podido observarlo previamente desde distintas posiciones y que implica: a) anticipación de un posible cambio de perspectiva dado un cambio de posición y b) anticipación de los cambios específicos en la apariencia del modelo dado un cambio de posición.

2. Un proceso de reconocimiento ${ }^{1}$ cuando el sujeto ha tenido la oportunidad de desplazarse previamente en torno al modelo, que no implica necesariamente ninguna de las dos condiciones a) y $b$ ), aunque es muy probable que la condición b) pueda estar presente en mayor o menor medida según la complejidad del problema, como veremos más adelante.

Según han mostrado algunos estudios (Masangkay et al., 1974; Flavell et al., 1978, 1981), los niños, desde los 5 años, están en posesión de ciertas reglas elementales que les permiten comprender, por ejemplo, que dos observadores pueden tener perspectivas diferentes de un mismo objeto, es decir, nuestra condición a). Estas reglas son una condición necesaria pero no suficiente para la resolución de tareas como las de coordinación de perspectivas. Comprender cómo se ve un objeto o grupo de objetos desde una perspectiva particular - condición b) - es una adquisición posterior que implica una serie de operaciones mentales en las que algunas relaciones se mantienen (como la distancia o la relación de intermediario), mientras que otras se modifican (derecha-izquierda, etc.).

En la Tabla 1 se recogen los resultados que se han obtenido en distintas investigaciones según el procedimiento utilizado (que llamaremos I y R). En general, los estudios realizados bajo la condición $\mathbf{R}$ encuentran menos errores egocéntricos y edades de adquisición más tempranas. Es posible que el menor número de respuestas egocéntricas en $R$ que en I se deba a que, como hemos señalado, bajo la condición $\mathrm{R}$ el sujeto tiene la oportunidad de comprobar que el modelo tiene una apariencia diferente cuando lo observa desde posiciones distintas. Incluso aceptando que ya desde los $s$ años los niños emplean algún tipo de regla semejante a la que hemos descrito como condición a), parece evidente que la utilizan con mayor frecuencia en $R$ que en $I$ para intentar dar respuestas no centradas en su propia perspectiva ${ }^{2}$.

Un hecho interesante es que con el procedimiento I ningún sujeto que no esté en posesión de un pensamiento operatorio resuelve la tarea. Por el contrario, la condición $\mathrm{R}$ arroja resultados mucho más dispares en cuanto a edades en las que los sujetos resuelven satisfactoriamente las tareas. La explicación no es dificil de encontrar. Las tareas que se 
TABLA $\mathbf{I}$.

Resultados en las tareas de perspectivas seguin el procedimiento utilizado: reconocimiento

(R) o inferencia (I). Entre paréntesis figuran las edades en las que disminuyen

significativamente los errores (tanto egocéntricos como no egocéntricos). Basado parcialmente en Eiser (1974) y completado con datos de otros estudios

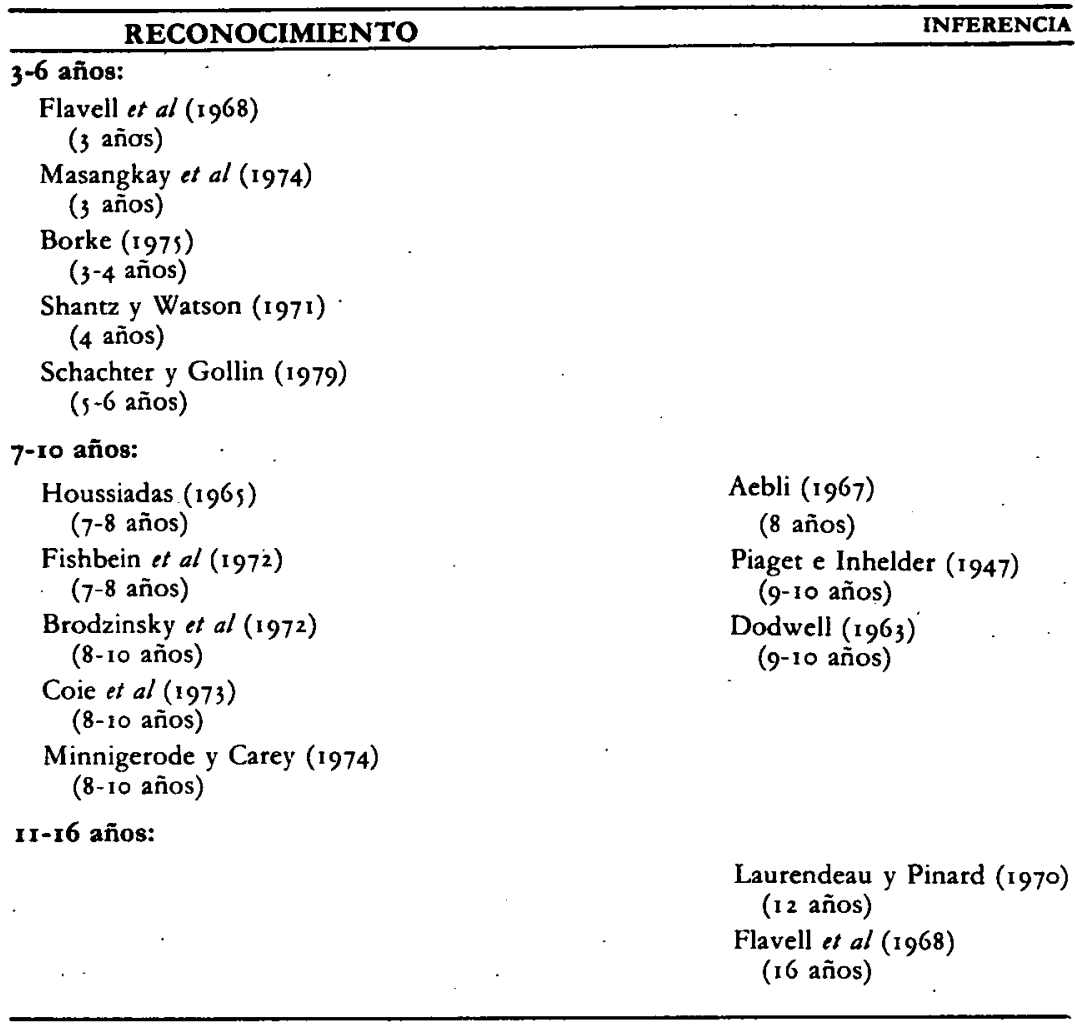

incluyen en, la categoria $\mathrm{R}$ muestran diferencias cruciales entre si, tanto en dificultad como en el tipo de habilidad que exige cada una. Analicemos, por ejemplo, una de las tareas de Flavell y colaboradores que niños de 2-3 años resuelven sin dificultad. El material de esta prueba consiste en una lámina con el dibujo de un perro en una de sus caras y el de un gato en la otra. El problema que se plantea al niño es el de averiguar qué animal ve el experimentador, sentado frente a él, cuando él está viendo el perro.

Nos parece interesante incluir la descripción de esta tarea, a pesar de su lejanísima relación con la prueba piagetiana, debido a que en más de una ocasión los resultados de Piaget e Inhelder han sido puestos en tela de juicio a partir de hallazgos como los de Flavell con esta tarea. Podríamos considerar en un sentido muy amplio que la prueba del perro y el gato es un problema de perspectivas. Es cierto que el niño tiene que "adoptar» la perspectiva del experimentador para saber que lo que está viendo es el gato. Pèro esta capacidad no requiere ningún tipo de coordinación de perspectivas ni, en consecuencia, transformación de relaciones espaciales (derecha-izquierda, delante-detrás, etc.), presentes en problemas como el de «Las Tres Montañas». El problema del perro y el gato tiene que ver más con la capacidad de comprender que un objeto permanece escondido para un observador y no para otro -capacidad que se desarrolla con la función simbólica - que con la habilidad de coordinar perspectivas. 
De hecho, la diferencia fundamental entre las tareas que se incluyen en ia categoria de reconocimiento radica, precisamente, en la presencia o no de un problema de coordinación de perspectivas propiamente dicho, es decir, de coordinación de relaciones interfigurales e intrafigurales. Mientras que en algunas de las experiencias de Flavell, Masangkay y otros, el niño tiene que demostrar simplemente su comprensión de que al cambiar de posición puede cambiar su perspectiva de un objeto, o que para ver la cara oculta de un objeto debe desplazarse 180 grados, tareas como las de Brodzinsky et al. (1972); Coie et al. (1973) y otros, exigen, además, que el sujeto se imagine exactamente las posiciones relativas de un grupo de objetos desde una perspectiva determinada. No deja de ser interesante el hecho de que cuando están en juego coordinaciones interfigurales e intrafigurales de modelos de cierta complejidad, los niños menores de 7-8 años son incapaces de resolver la tarea, incluso con el procedimiento R. Parece, pues, que al aumentar la complejidad del modelo disminuye también la probabilidad de una solución por reconocimiento.

$\mathrm{Y}$, entonces, el sujeto debe realizar el mismo tipo de operaciones mentales que con el procedimiento I (a excepción de la condición a), descrita anteriormente), es decir, anticipar los cambios específicos en la apariencia del modelo desde una perspectiva particular. Y esta habilidad parece depender de un funcionamiento operatorio.

En resumen, según los estudios que hemos analizado, el procedimiento $\mathrm{I} \cdot \mathrm{O} \mathrm{R}$ parece afectar fundamentalmente a lo que se denominan errores egocéntricos, es decir, respuestas centradas en el propio punto de vista del sujeto más que a la habilidad para coordinar las distintas perspectivas de un modelo. El desfase cronológico que se observa en la resolución de este tipo de problemas (tanto en I como en R) puede explicarse por el grado de familiaridad de los objetos utilizados y la complejidad del modelo (véase apartado anterior).

\section{Segun la respuesta: identificación o construcción}

Las tareas de perspectivas varían también según el tipo de respuesta que debe dar el sujeto y que puede consistir en una identificación o en una construcción de la perspectiva requerida. En el primer caso, el niño debe elegir entre una serie de representaciones bidimensionales o tridimensionales de las distintas perspectivas del modelo aquella que considera correcta. El segundo tipo de respuesta consiste en construir la perspectiva pedida con ayuda de elementos que representan a cada uno de los objetos del modelo. Piaget e Inhelder emplearon ambas técnicas en el estudio de las perspectivas observando reacciones homogéneas en los sujetos de un mismo nivel cognitivo, cualquiera que fuera la técnica adoptada.

La mayoría de las investigaciones posteriores han retomado casi exclusivamente la técnica de identificación. Este hecho no obedece aparentemente a ninguna causa específica, pues hasta la investigación de Flavell et al. (ig68), no se pusieron de manifiesto las dificultades diferenciales de ambas. Posteriormente, Rubin (1973, 1974), Hoy (1974) y Pufall (1975) emplearon también el método de construcción, pero pocos más son los autores que lo han incorporado a sus investigaciones. 
Tanto Flavell como Hoy encontraron que los sujetos tenian más dificultades en construir las perspectivas que en identificarlas. Sin embargo, Pufall (1975) estudió la construcción de perspectivas en niños de, a 6 años sin observar mayores dificultades que las propias de la edad. En una investigación reciente (Enesco, 1982) hemos podido comprobar las ventajas de la técnica de construcción sobre la de identificación a la hora de estudiar la coordinación de perspectivas. Hay dos razones cruciales por las que la primera técnica resulta mucho más ventajosa $e$ inequivoca que la segunda. Una de ellas es la interpretación de la respuesta del sujeto y la otra se refiere a las estrategias que utiliza para resolver el problema.

En el procedimiento de identificación nos enfrentamos con el siguiente problema respecto a la interpretación de la respuesta. En este caso, el sujeto debe elegir uno de los estímulos de elección que le presenta el experimentador. Esta elección puede considerarse bien una respuesta correcta, si se trata de la perspectiva apropiada; bien incorrecta, si elige cualquier otra cosa. Además, según la respuesta incorrecta, ésta puede clasificarse como egocéntrica (cuando el sujeto elige la propia perspectiva habiéndosele pedido otra) o no egocéntrica (cualquier perspectiva que no sea la correcta ni la propia). De acuerdo con esto, la probabilidad de elegir al azar una "respuesta» correcta es la misma que la de elegir una egocéntrica. A la vez; la probabilidad de elegir aleatoriamente respuestas incorrectas no egocéntricas aumenta con el número de estímulos de elección.

Probablemente, una importante fuente de confusión respecto a la existencia o no de egocentrismo (recordemos que muchos autores hablan de un porcentaje "despreciable» de errores egocéntricos) sea sencillamente el número de estímulos de elección, una variable que no ha sido controlada habitualmente. Si es superior a tres (el número de categorías de respuesta) y el niño elige al azar, la probabilidad de un error no egocéntrico

$$
\text { ( } \left.\frac{\text { núm. de estímulos }-2}{\text { núm. de estímulos }}\right)
$$

es mayor que la de uno egocéntrico

$$
\left(\frac{1}{\text { núm. de estímulos }}\right) \text {. }
$$

Teniendo en cuenta que en la mayoría de las investigaciones se han utilizado de 4 a ro estimulos, la probabilidad de error no egocéntrico no es despreciable.

Por otra parte, no sería posible diseñar un test estadístico destinado a validar o refutar la hipótesis de que el niño elige al azar sus respuestas cuando comete errores no egocéntricos, dado que el número de observaciones que se suelen hacer es de tres ( 90,180 y 270 grados). Así pues, los errores no egocéntricos no pueden interpretarse inequivocamente. Por otra parte, la valoración de las respuestas correctas y egocéntricas es menos ambigua cuanto mayor sea el número de estimulos de elección. Ahora bien, existen pruebas de que la proporción de errores aumenta significativamente cuando se utilizan más de 
ocho estímulos de elección (Fehr, 1978) y, probablemente, con ello lo que están aumentando son las respuestas aleatorias.

La segunda razón de peso a favor de la técnica de construcción tiene que ver con la conducta del sujeto durante la resolución de la tarea. Con la técnica de construcción no obtenemos únicamente una respuesta, correcta o incorrecta, como ocurre con la identificación. El proceso que conduce a la respuesta final indica qué tipo de relaciones espaciales considera el sujeto prioritariamente, qué tipo de errores persisten y, en general, detecta niveles de actuación mucho más sutiles que escapan al proceso de identificación. Nosotros hemos encontrado, además, que utilizando la técnica de construcción con elementos tridimensionales (es decir, objetos idénticos a los del modelo), el porcentaje de respuestas egocéntricas es muy bajo, al menos desde los 6 años. La explicación que hemos dado a estos resultdos es que, cuando el sujeto tiene que construir una perspectiva diferente a la suya con objetos reales y no con fotos o dibujos, hay relaciones espaciales elementales (como las de proximidad-lejania) cuyas transformaciones puede intuir con bastante precocidad. Sin embargo, con la técnica de identificación, al sujeto se le impide realizar los tanteos de proximidadlejanía de los objetos respecto al observador, los de orientación de cada objeto en relación con el observador y de los objetos entre sí, etc. Estos rudimentos de relaciones espaciales con los que cuenta el niño son, a nuestro juicio, obstaculizados cuando lo que se le pide es que identifique una perspectiva determinada a partir de sus representaciones en plano (donde, obviamente, sólo están presentes las relaciones izquierda-derecha y delante-detrás, pero no las de proximidad).

\section{Seguin la perspectiva: tarea perceptiva o tarea conceptual}

Por lo general, se da por hecho que las dificultades en la adopción de perspectivas se presentan sólo cuando el sujeto debe representarse un punto de visto ajeno al propio.

Hay, sin embargo, pruebas de que la habilidad para reproducir el propio punto de vista no es tan precoz y general como se suponía. A este segundo tipo de tarea la denominamos perceptiva porque son, fundamentalmente, factores perceptivos más que conceptuales los que están en juego, a diferencia del problema de imaginarse una perspectiva distinta.

Nigl y Fishbein (1974) estudiaron el desarrollo de la comprensión perceptiva y conceptual de las relaciones espaciales izquierda-derecha, delante-detrás y cerca-lejos en niños de 4 a 11 años de edad. Aunque para todos los sujetos fue más dificil la tarea de inferir otras perspectivas, los errores en la reproducción de la propia perspectiva no desaparecieron hasta los ocho años y medio. Un dato interesante es que los sujetos reproducían, en la tarea conceptual, las mismas dificultades que habian tenido en la tarea perceptiva en edades anteriores. En ambos casos, la relación espacial que se dominaba en primer lugar era la de proximidad-lejanía y la más difícil la relación izquierda-derecha, resultados comúnmente confirmados en la investigación sobre perspectivas.

Estos hallazgos, más que confirmar la existencia de una fase de 
egocentrismo, ponen de manifiesto dificultades ligadas estrictamente al proceso de extracción de la información perceptiva y la puesta en correspondencia de dos imágenes perceptivas. En términos piagetianos, la tarea perceptiva se podría definir como la construcción de una imagen estática, puesto que el sujeto debe construir una imagen del modelo tal y como lo percibe. Sin embargo, la tarea conceptual exige la construcción de imágenes anticipatorias que, dentro del modelo piagetiano de desarrollo, caracterizan el funcionamiento operatorio. Incluso con referencia a la propia teoría piagetiana, las dificultades en ambos tipos de tarea podrian explicarse de acuerdo con el desarrollo de las imágenes mentales, sin necesidad de acudir a la interpretación en términos de egocentrismo.

Por último, respecto a las dificultades inherentes a la propia tarea conceptual, según que el sujeto deba adoptar perspectivas frontales ( 180 grados), laterales (90 y 270 grados) u oblicuas (4s y 135 grados), los hallazgos a este respecto son contradictorios. Eiser (1974), por ejemplo, observó que las rotaciones de 180 grados eran significativamente más fáciles para todos los sujetos (de 6-7 a 8-7 años), seguidas de las oblicuas $y$, en último lugar, las laterales. Por el contrario, Nigl y Fishbein (1974), Pufall (1975) y otros autores han observado mayores dificultades en la adopción de una perspectiva frontal que de las laterales.

La investigación de Pufall aporta ciertos resultados interesantes que pueden clarificar parte de estas contradicciones. Pufall encontró un porcentaje elevado de errores tanto en la perspectiva de 180 grados como en las laterales, cuando el modelo constaba de objetos simétricos respecto a sus lados y respecto a sus posiciones (por ejemplo, dos árboles situados simétricamente respecto a todos los ejes) (véase figura I). Cuando se rompia la simetría de posición y orientación, los

a

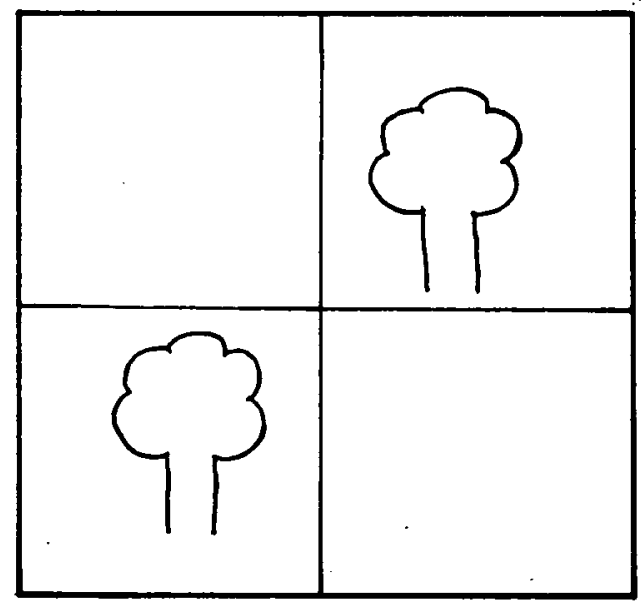

Figura i

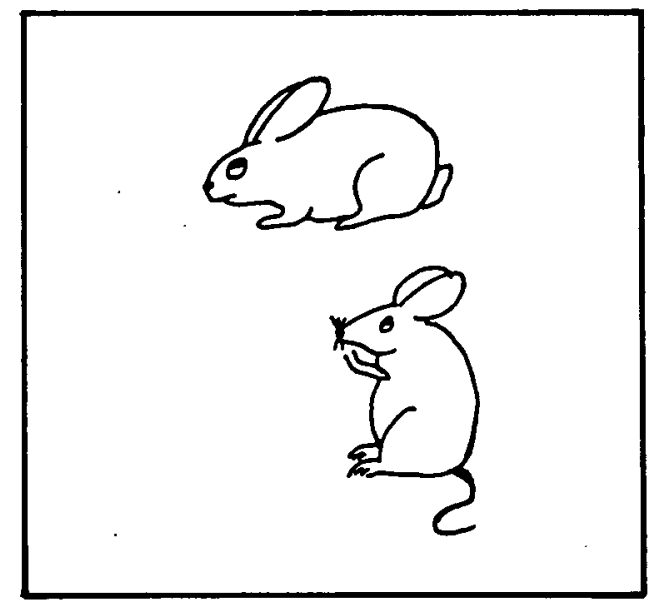

a) Modelo con dos objetos simétricos respecto a sus lados y respecto a sus posiciones.

b) Modelo con objetos asimétricos y en una disposición asimetrica.

En a) la inversión de 180 grados no afecta en absoluto la apariencia del modelo. En b) esta inversión transforma las relaciones de proximidad-lejanía del observador respecto a los animales (o, lo que es lo mismo, las relaciones «delante-detrás» de éstos respecto al observador) y transforma las orientaciones derecha-izquierda de los animales.

En a) los giros de 90 y 270 grados afectan tan sólo a las relaciones derecha-izquierda de los árboles (superior e inferior). En b) estos giros implican transformaciones múltiples de las posiciones y orientaciones de los animales respecto al observador. 
errores disminuian considerablemente en las perspectivas laterales, aunque menos en la de 180 grados. En esta última, la mayoria de los errores fueron de carácter egocéntrico.

Pufall sugiere que las relaciones asimétricas, sean de posición o respecto a los distintos lados del objeto, provocan un desequilibrio en el niño que lo lleva a descartar soluciones egocéntricas y a explorar activamente las relaciones espaciales implícitas en los objetos. En otras palabras, cuando la información diferencial entre objetos o intra-objeto disminuye, la conducta egocéntrica del sujeto aumenta, probablemente, porque le resulta más difícil abstraer una información que no está contenida ni en los objetos ni en sus posiciones relativas. Según esta interpretación, la mayor dificultad de la perspectiva frontal, observada en varias investigaciones, se debería a este factor de simetría, puesto que una rotación de 180 grados invierte todas las posiciones y orientaciones relativas derecha-izquierda, delante-detrás, cerca-lejos, conservando la simetría respecto al eje del propio sujeto. Según las posiciones de los objetos, la perspectiva lateral puede ser también simétrica respecto a la del sujeto. En esos casos, según Pufall, las dificultades son tantas como en la perspectiva de 180 grados.

Aunque la interpretación de Pufall no explica resultados como los de Eiser (1974), parece bastante plausible que, al menos en algún momento del desarrollo, este factor de simetría obstaculice la tarea y refuerce lo que Piaget ha denominado centración, conduciendo a una conservación de las relaciones egocéntricas. Sin embargo, muy probablemente debamos buscar otras explicaciones para entender los procesos subyacentes a la tarea conceptual.

Si partimos del hecho de que el estudio de Pufall se ralizó con niños de 5 a 6 años y el de Eiser con sujetos algo mayores (6-7 a 8-7 años), por poner sólo dos ejemplos, podríamos suponer como hipótesis que las estrategias cognitivas de los sujetos en la resolución de tareas de perspectivas varían con la edad y que un cambio de estrategia puede implicar un cambio en las dificultades relativas de la tarea conceptual.

Supongamos, con Huttenlocher y Presson (1973), que para resolver este tipo de problema el sujeto puede utilizar distintas estrategias. Una de ellas sería girar mentalmente el modelo desde la perspectiva pedida hasta la posición del sujeto y luego «leer» la imagen resultante. La segunda consistiria en desplazarse mentalmente hasta la posición requerida y «leer» la imagen desde esa perspectiva. Por último, el sujeto podría también aplicar sistemáticamente un tipo de regla de inversiones o giros de cada elemento del modelo. Las dos primeras estrategias implican una solución predominantemente "visual» (se entiende, de imagen visual) mientras que la tercera tiene un carácter más lógico. Actualmente, no se sabe más que a título de hipótesis si en todas las edades se utilizan las mismas estrategias o si los sujetos aplican un tipo u otro de estrategia dependiendo de la perspectiva a adoptar o del problema que se les plantea ${ }^{3}$.

Una hipótesis bastante plausible es que la tercera estrategia no es utilizada por los niños pequeños y que se incorpora con posterioridad a las otras dos (Enesco, 1982). La situación más sencilla a la que puede aplicarse esta estrategia es la perspectiva de 180 grados, puesto que el sujeto debe aplicar siempre la misma regla, a saber, la de invertir todas las relaciones espaciales (de posición y de orientación). En otras 
palabras, todo lo que está a la derecha, respecto al observador, pasa a estar a la izquierda; todo lo que está "cerca" pasa a estar «lejos", etc. En las perspectivas laterales ( 90 y 270 grados) algunas relaciones se invierten y otras se conservan, es decir, cada elemento del modelo sufre sólo una transformación de posición (izquiera-derecha $o$ delante-detrás/cerca-lejos), además de los cambios de orientación presentes también en las rotaciones de 180 grados.

Podría argüirse que también se puede aplicar una regla muy simple para las perspectivas laterales. Por ejemplo, en la figura 2 vemos que cualquiera de estas perspectivas se resuelve desplazando cada elemento (en la dirección de las manillas del reloj para la rotación de 90 grados y en la dirección opuesta para la de 270 grados) al siguiente cuadrante más próximo. No obstante, esta regla, tan elemental para cualquier persona familiarizada con problemas de giros y rotaciones, puede resultar menos intuitiva que la regla de inversiones presente en la rotación de 180 grados $^{4}$. Mientras que esta última puede formularse en

Figura 2

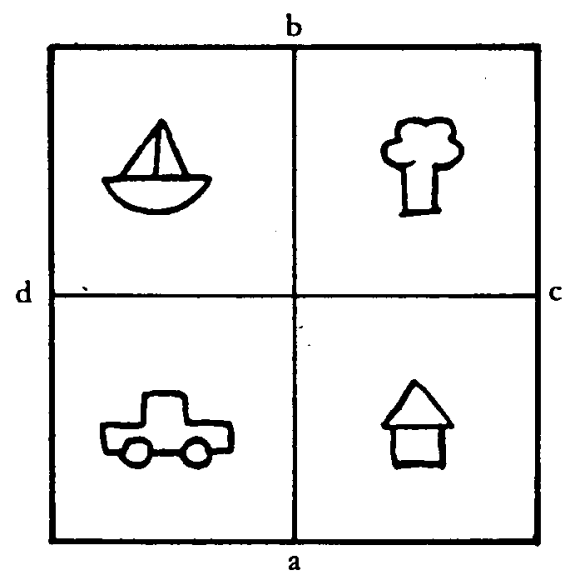

Modelo (o grados)

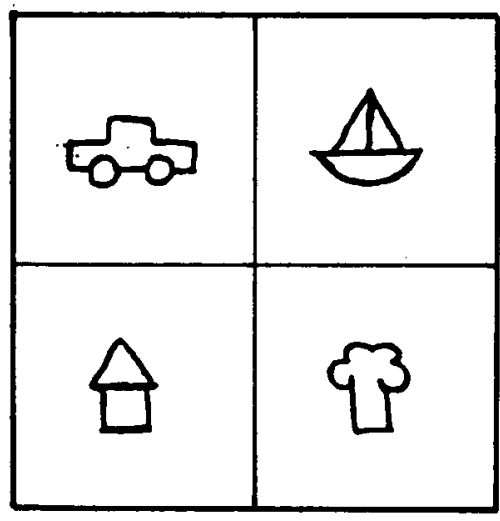

Giro de 90 grados*

[perspectiva desde c)]

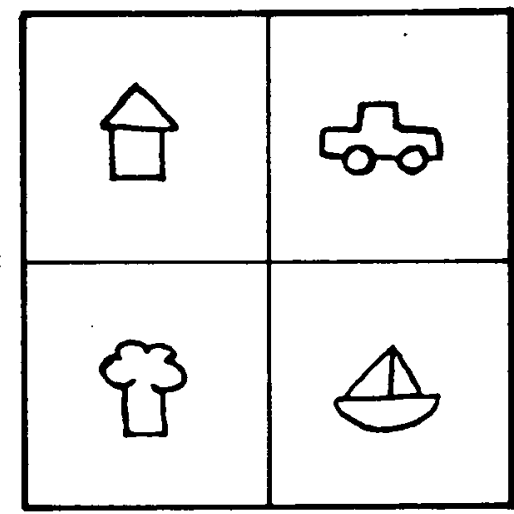

Giro de 180 grados

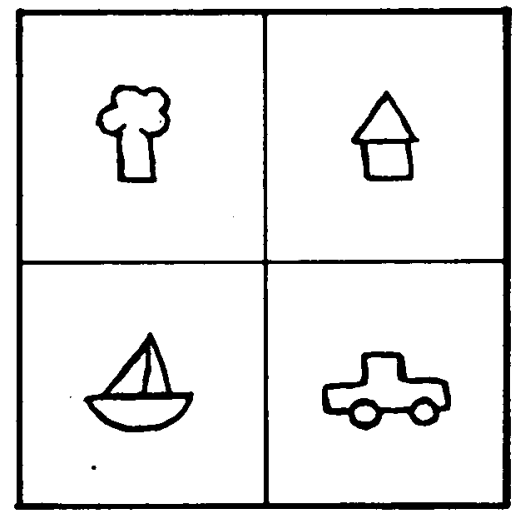

Giro de 270 grados * [perspectiva desde d)]

* Para simplificar las orientaciones de los objetos se han dejado idénticas a como aparecen en el modelo aun cuando éstas, lógicamente, se modifican en función de la perspectiva. 
una estrategia de (invertir todo» (posiciones y orientaciones), en la anterior la única formulación posible contendría el propio concepto de giro de 90 grados. Así pues, la tercera estrategia, que resulta muy económica en la perspectiva de 180 grados, puede no serlo en las demás y es probable que en estos otros casos la solución visual sea más frecuente.

Esta hipótesis, junto con la de Pufall sobre el efecto del factor de simetría, explicaría que hasta una determinada edad los sujetos cometan más errores en la perspectiva de 180 grados al carecer de esta regla de inversiones y que, posteriormente, se inviertan las dificultades relativas de la perspectiva frontal y de las laterales y oblicuas en las que la solución visual es más probable, pero también más sujeta a errores.

\section{EGOCENTRISMO FRENTE A PERSPECTIVISMO: CONCLUSIONES}

El habernos detenido con cierto detalle en buen número de investigaciones nos ha servido para poner de manifiesto dos caracteristicas de la investigación sobre perspectivas espaciales. En primer lugar, la variabilidad entre las tareas en cuanto a las edades en que se resuelven. En algunas, niños desde los 3 y 4 años son capaces de adoptar la perspectiva de otra persona, mientras que, en otras, los sujetos no son capaces de reproducir correctamente una perspectiva ajena hasta por lo menos los 12 ó 13 años. En segundo lugar, la variabilidad en los tipos de errores que cometen los sujetos cuando tienen que representarse perspectivas ajenas. Varios autores han observado la existencia de errores egocéntricos (reproducción del propio punto de vista cuando se trata de imaginar otra perspectiva) e incluso una persistencia de éstos hasta edades muy avanzaas (Laurendeau y Pinard, 1970; Flavell et al., 1968). Sin embargo, otros estudios no encuentran que sean tan frecuentes ni que exista una etapa de egocentrismo en el desarrollo de las perspectivas (Fishbein et al., 1972; Coie et al, 1973). Flavell y sus colaboradores (1968) comprobaron esta variabilidad entre tareas, tanto en la edad de adquisición como en la presencia o no de egocentrismo, al administrar una bateria de pruebas diferentes de adopción de perspectivas a sujetos de 3 a 16 años.

El hecho de que los niños pequeños comprendan la existencia de otras perspectivas aun cuando no sean capaces de saber cómo son exactamente, como han puesto de manifiesto algunos estudios, constituye un desafio a la tesis piagetiana sobre el egocentrismo infantil. Recordemos cómo definen Piaget e Inhelder las conductas egocéntricas caracteristicas del subestadio IIA:

El sujeto se muestra incapaz de comprender que observadores diferentes verán el macizo (de montañas) según perspectivas distintas y parece imaginarse que su propio punto de vista es el único posible (1947, pág. 248).

En lugar de representarse las perspectivas que corresponden a las diversas situaciones, el niño considera el punto de vista en el que está situado momentáneamente como el único posible y no consigue deducir las transformaciones originadas por los cambios de posición del observador (op. cit., pág. 2 50).

Las críticas a la concepción piagetiana de egocentrismo se han basado, fundamentalmente, en definiciones como las que citamos en los dos párrafos anteriores. Si, efectivamente, los niños pequeños de lo que 
carecen es de la habilidad para anticipar la apariencia de un objeto o grupo de objetos desde perspectivas diferentes pero no de la comprensión de la existencia de éstas (Flavell et al., 1981, etc.), entonces el concepto de egocentrismo debería, en el mejor de los casos, ser reformulado.

Llegados a este punto, creo que es obligado decidirse por una interpretación de las dificultades inherentes a la adopción de perspectivas. Mi opinión es que la misma variabilidad de los resultados es una buena razón para enfocar el problema desde una perspectiva distinta. Y esta perspectiva tiene que ver con una distinción básica, propuesta por Turiel (1983), entre los métodos que utilizamos las personas para obtener información sobre el mundo que nos rodea y el sistema conceptual implicado en dicha información. Adoptar la perspectiva de una persona, es decir, ponerse simbólicamente en su lugar, es un método para obtener información de lo que está viendo, de lo que piensa o de lo que siente esa persona. El método, como tal, puede aplicarse a numerosas situaciones y en relación con distintos sistemas conceptuales (espaciales, psicológicos, sociales...). Obviamente, el método en si mismo no puede estudiarse en su forma pura, pues al situarnos en el punto de vista del otro lo hacemos siempre con referencia a algo, su percepción visual, sus sentimientos, su conocimiento, etc. Pero esto no significa que la actividad de adopción de perspectivas y los conceptos espaciales o nuestro conocimiento de la psicología del otro sean lo mismo. Si un sujeto fracasa cuando se le pide que imagine lo que está viendo otra persona o su estado emocional, no tiene que ser necesariamente porque es incapaz de situarse en puntos de vista ajenos. Quizá su problema es el propio sistema conceptual implicado y su éxito o fracaso dependa del nivel que haya alcanzado el sujeto en ese dominio particular (Turiel, op. cit., págs. $71-73)$.

Por los resultados que hemos comentado a lo largo de este artículo y por otras investigaciones sobre adopción de perspectivas (no sólo espaciales) (Ford, 1979) parece evidente que esta habilidad aparece hacia los 3 ó 4 años, dependiendo del desarrollo del pensamiento representacional. Pero aunque el niño manifieste una competencia precoz en la adopción de perspectivas, su actuación varía de acuerdo con el tipo de tarea que debe realizar.

Queda, por tanto, poco lugar para una concepción del egocentrismo como una etapa en la que el sujeto considera su punto de vista como el único posible. Flavell (1974) ha propuesto alternativamente a la tesis piagetiana que la tendencia del desarrollo iría hacia una habilidad creciente para determinar perspectivas ajenas con mayor precisión y no desde el egocentrismo hasta el perspectivismo. De hecho, este mismo autor ha observado repetidas veces que los niños conocen la existencia de otras perspectivas mucho antes de saber exactamente qué es lo que ve otro observador.

Una conclusión de este artículo es que el concepto de egocentrismo, entendido como actitud cognitiva del sujeto, resulta inoperante. Más que de egocentrismo debería hablarse de errores egocéntricos ligados a situaciones especificas pero no ligados a la edad ni necesariamente a un determinado nivel cognitivo. Es probable, como han sugerido 
algunos autores (Aebli, 1967: Shantz y Watson, 1970, y otros), que el egocentrismo constituye una solución «alternativa» a un problema demasiado dificil para el sujeto. Sin embargo, habria que preguntarse por qué el sujeto acude a este tipo de respuesta cuando carece de una solución para el problema.

Es probable que los errores que comete el sujeto, sean o no egocéntricos, estén relacionados con otros factores cognitivos además de los espaciales. Pocos estudios hay al respecto pero en uno de ellos (Liben y Belknap, 198I) se ha podido demostrar que factores como el realismo intelectual interfieren en la resolución de estas tareas. En este caso, no seria la incapacidad del niño para diferenciar entre su propia experiencia visual y la de otros lo que le llevaria a cometer errores, sino su incapacidad para distinguir entre su propio conocimiento y su propia experiencia visual del modelo.

Pero, en última instancia, cualquiera que sea el origen de los errores egocéntricos, la habilidad de adopción de perspectivas en si misma no es lo que está en juego en todas estas tareas, sino más bien los diferentes sistemas conceptuales que implica cada una de ellas.

\section{Notas}

1 Como se describe en la página 89 y siguientes, la tarea de perspectivas puede consistir en una identificación o en una construcción de las perspectivas. Aunque en el primer caso podemos hablar de reconocimiento (cuando el sujeto ha tenido la oportunidad de desplazarse previamente en torno al modelo) no ocurre lo mismo en la segunda situación, en la que el sujeto debe construir la perspectiva requerida a partir de elementos que representan a los del modelo, y no simplemente uelegir» entre distintas representaciones del modelo aquella que considera la perspectiva correcta. La técnica de construcción no permite una solución por reconocimiento. Al hablar de un proceso de reconocimiento frente a uno de inferencia nos referimos exclusivamente a los estudios que han utilizado la técnica de identificación.

2 Hay que decir que aunque Piaget e Inhelder utilizaron fundamentalmente el procedimiento 1 también introdujeron algunas condiciones semejantes al procedimiento $R$, sin encontrar diferencias sustanciales en las respuestas de los niños. Es decir, observaron el mismo tipo de errores (tanto egocéntricos como no), y edades de adquisición semejantes con ambos procedimientos. En relación con las respuestas egocéntricas, los autores ginebrinos interpretaron estos resultados como una prueba de que el egocentrismo del niño es «impermeable» a la experiencia, incluso cuando ésta contradice la solución dada por el niño. No obstante, este resultado no se encuentra en otras investigaciones semejantes en las que se controlan mejor los efectos de las condiciones de aplicación de la prueba.

3 Algunos hallazgos como los de Huttenlocher y Presson (1973) apoyan la hipótesis de que los sujetos pueden utilizar estrategias diferentes dependiendo de cómo se les plantee el problema de las perspectivas. Estos autores observaron que cuando se les pedía explícitamente a los sujetos (niños de 8 a 10 años) que rotaran mentalmente el modelo hasta su posición, éstos cometían, por lo general, menos errores que cuando debian desplazarse mentalmente alrededor del modelo, lo que puede interpretarse como una prueba de la presencia de estrategias diferentes.

4 Informalmente hemos comprobado con algunos adultos no familiarizados con este tipo de problemas que éstos tienen muchas más dificultades para llegar a inferir la regla de los giros de 90 grados que la de inversiones.

La solución más probable entre estos adultos era la que hemos denominado solución "visual" (por desplazamiento mental hasta la posición requerida o por rotación mental del modelo) para los giros de $9^{\circ}$ grados mientras que en la de 180 grados la mayoria utilizaba también la estrategia de cinvertir todon, según los informes de los propios adultos al finalizar la tarea

\section{Resumen}

Uno de los conceptos polémicos de la teoria piagetiana, el egocentrismo, es revisado en este articulo en el contexto de las tareas de adopción de perspectivas espaciales. Algunos autores ban encontrado un elevado porcentaje de respuestas egocéntricas en niños pequeños en este tipo de tareas. Otros, sin embargo, observan posos errores egocéntricos en todas las edades sin encontrar una etapa de egocentrismo en el desarrollo espacial, tal y como la describe la teoria piagetiana. Estas discrepancias se deben, en parte, a las diferencias metodologicas en el estudio de la babilidad para adoptar otras perspectivas. Además de analizar los aspectos metodológicos de la investigación, se propone en este articulo una reinterpretación del concepto de egocentrismo ligada a la distinción, propuesta por Turiel (1983), entre la adopción de perspectivas ajenas - como método para obtener información - y el sistema conceptual implicado en dicba información. 


\section{Summary}

This paper reviews egocentrism, a polemical concept of Piagetian theory, in the context of spatial perspective-taking tasks. Some autbors bave reported a bigh percentage of egocentric responses in young children in such problems, whereas otbers found few egocentric errors at all ages and did not identify the stage of egocentrism in spatial development, which is described in Piagetian theory. These discrepancies are partly due to metbodological inconsistencies in the study of spatial perspective-taking ability. Besides analysing such metbodological aspects, this paper favours a reinterpretation of egocentrism tied to the distinction, proposed by Turiel $(1983)$, between perspective-taking ability -as a metbod for gathering information- and the conceptual system involved in such information.

\section{Résumé}

Dans cet article nous revisons la notion d'égocentrisme, une des plus polémiques de la théorie Piagetienne, du point de vue des problemes de perspectives spatiales. Quelques auteurs ont trouvé un grand pourcentage de réponses égocentriques chez les petits enfants dans ces sortes de tâches, tandis que d'autres ne trouvent qu'un nombre petit d'érreurs égocentriques à tous les ages, sans reconnaître l'étage d'égocentrisme décrite par Piaget dans le développement de lespace. Ces discordances peuvent être expliquées, en partie, par les différences métbodologiques qu'on observe dans l'étude des perspectives spatiales. Dans cet article nous éxaminons les aspects méthodologiques de la recherche en proposant une réinterprétation de la notion d'égocentrisme liée à la distinction, faite par Turiel (1983), entre l'babilité pour se placer dans de perspectives différentes -entendue comme une méthode pour obtenir information et le systeme conceptuel impliqué dans cette information.

\section{Referencias}

AEBI.I, H.: «Egocentrism: not a phase of mental development but a "substitute solution" for an insoluble task». Pedag. Europ., 3, págs. 97-103, 1967.

BORkE, H.: «Piaget's mountains revisited: changes in the egocentric landscape». Developmental Psycbology, 11,2 , págs. 240-243, 1975 .

Brodzinski;, D. N.; JACKson, J. P., y OVERTon, W. F.: "Effects of perceptual shielding in the development of spatial perspectives). Child Development, 43, 3, págs. 1014-1016, 1972.

Coif, J. D.; Costanzo, P. R., y FARnil.., D.: "Specific transition in the development of spatial perspectives-taking ability». Devel. Psycbl., 9, 2, págs. 167-177, 1973.

Cox, M. V.: "The other observer in a perspective task". British Journal of Educational Psychology, 4s, págs. $83-85$, 1975 .

Cox, M. V.: "Perspective ability: the relative difficulty of the other observer's viewpoints". Journal of Experimental Child Psychology, 24, págs. 2\$4-259, 1977.

Deregowski, J. B.: "Orientation and perception of pictorial depth». International Journal of Psychology, 6 , págs. IIt-114, 1971 .

EISER, C.: «Recognition and inference in the coordination of perspectives». British Journal of Educat. Psychol., 44, 3, págs. 309-312, 1974 .

EISER, C.: "Questions children ask about spatial arrays. An analysis of the processes involved in coordinating perspectives". British Journal of Educationsl Psychology, 46, págs. 203-211, $197^{6}$.

Ei.konin, D. B.: Psicologia del juego. Madrid, Pablo del Río, 1980 y Visor-Aprendizaje, 1989.

ENESCo, I.: El desarrollo de conceptos espociales: Un estudio transcultural. Tesis doctoral. Universidad Complutense de Madrid, 1982.

FEHR, L. A.: «Methodological inconsistencies in the measurement of spatial perspective taking ability: A cause for concern». Human Development, 21, (5-6), págs. 302-315, 1978.

FISHBEIN, H. D.; LEW'1S, S., y KEIFFER, K.: «Children's understanging of spatial relations: coordinating of perspectives". Devel. Psychol., 7, 1, págs. 21-23, 1972.

Fi.AVEl.I., J. H.: "The development of inference about others». En T. MisheI. (Ed.): Understandin otber persons, Blackwells, Oxford, 1974.

Flavei.l, J. H.; Botkin, P. A.; FRY, C. L.; WRIGHT, J. W., y JARvis, P. E.: The development of the role-taking and communication skills in children, Wiley, Nueva York, 1968.

Fi.AVEı.ı, J. H.; OMASON, O., y LATHAM: «Solving spatial perspective-taking problems by rule versus computation: A developmental study». Develop. Psychol., 14, s, págs. 462-473, 1978.

Fi.avei.l, J. H.; Finvel.I., E. R.; Green, F. L., y Wil.cox, S. A.: «The development of three spatial perspective-taking rules». Child Development, $\{2,1$, págs. 396-358, $198 \mathrm{I}$

FORD, M. E. (1979): "The construct validity of egocentrism", Psycbological Bolletin, 1979, 86, 1169-88.

GARner, J., y Pi.ANT, E. L.: «On the measurement of egocentrism: A replication and extension of Aebli's findings". Brit. Journ. of Educat. Psycbol., 42, págs. 79-8 $3,1972$.

GiBSON, E. J.: Principles of perceptual learning and development. Appleton-Century-Crofts, Nueva York, 1969.

Hor, F. A.: «Predicting another's visual perspective: A unitary skill?». Develop. Psycbol., 10, pág. 462, 1974.

HuDSon, W.: “Cultural problems in pictorial perception». South African Journal of Sciences, s8, págs. $189-195$, 1962.

LAurendedu, M., y PINARD, A.: The development of the concept of space in child, Intern. University Press, Nueva York, 1970.

Hutteniocher, J., y Presson, C.: "Mental rotation and the perspective proble». Cognitive Psycbology, 4, 277-299, 1973

LIBEN, L., y BEI.KNAP, B.: «Intelectual realism: Implications for investigations of perceptual perspectivetaking". Cbild Develop., 52, págs. 921-924, 1981 . 
Masagkay, Z.; MCCiuskey, C. W.: Sims-KNight, J-; Vaugu, B. E., y F!.tiei.1, J. H.: "The early development of inferences about the visual percepts of others". Child Develop., 45, págs. 357+366, 1974.

MinnigeroDE, F. A., y CAREY, R. N.: «Development of mechanisms underlying spatial perspectives». Child Develop., 45, págs. 496-498, 1974.

Nigl, A. J., y FishbEIN, H. D.: «Perception and conception in coordination of perspectives». Develop. Psycbol., 10, 6, págs. 858-866, 1974.

Piaget, J., e Inhei.der, B.: La représentation de l'espace chez l'enfant. P. U. F., Paris, 1948.

Phinney, J. S., y Nummedi., S. G.: «Effects of left-right and position reversals on spatial perspective taking in young children». Perceptual \& Motor Skills, \$8 (1), págs. 223-227, 1979.

Pufal.J., P. B.: "Egocentrism in spatial thinking: It depends on your point of view". Develop. Psycbol., I, 3, págs. $297-303,1975$.

Rubin, K. H.: "Egocentrism in childhood. A unitary construct». Child Develop., 44, págs. 102-1 10, 1973.

SCHACHTER, D., y Gol.I.IN, E. S.: "Spatial perspective taking in young children». Journal of Experimental Child Psychology, 27 (3), págs. 467-478, 1979.

SHANTZ, C. U., y WATSON, J. S.: "Spatial abilities and spatial ego-centrism in the young child». Child Developm., 42, págs. $171-181,1981$.

TURIEI., E.: The development of social knowledge, Cambridge University Press, Cambridge. 